\title{
Identification of TFB5, a new component of general transcription and DNA repair factor IIH
}

\author{
Jeffrey A Ranish ${ }^{1}$, Steven Hahn ${ }^{2}$, Yu Lu ${ }^{1,3}$, Eugene C Yi ${ }^{1}$, Xiao-jun Li ${ }^{1}$, Jimmy Eng ${ }^{1}$ \& Ruedi Aebersold ${ }^{1}$
}

We previously described the use of quantitative proteomics to study macromolecular complexes ${ }^{1}$. Applying the method to analyze a yeast RNA polymerase II preinitiation complex, we identified a new 8-kDa protein, encoded by the uncharacterized open reading frame YDR079c-a, as a potential new component of the preinitiation complex. Here we show that YDR079c-a is a bona fide component of polymerase II preinitiation complexes and investigate its role in transcription. YDR079c-a is recruited to promoters both in vivo and in vitro and is required for efficient transcription in vitro and for normal induction of GAL genes. In addition, YDR079c-a is a core component of general transcription and DNA repair factor $\mathrm{IIH}$ and is required for efficient recruitment of TFIIH to a promoter. Yeast lacking YDR079c-a grow slowly, and, like strains carrying mutations in core TFIIH subunits, are sensitive to ultraviolet radiation. YDR079c-a is conserved throughout evolution, and mutations in the human ortholog account for a DNA repair-deficient form of the tricothiodystrophy disorder called TTD-A ${ }^{2}$. The identification of a new, evolutionarily conserved, core TFIIH subunit is essential for our understanding of TFIIH function in transcription, DNA repair and human disease.

\section{a}

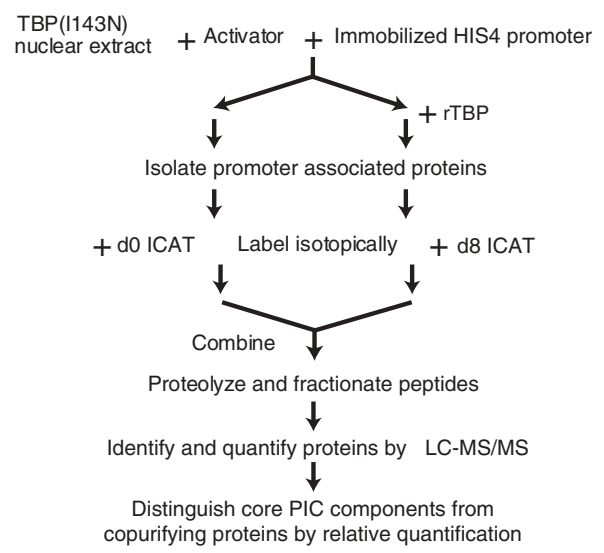

b

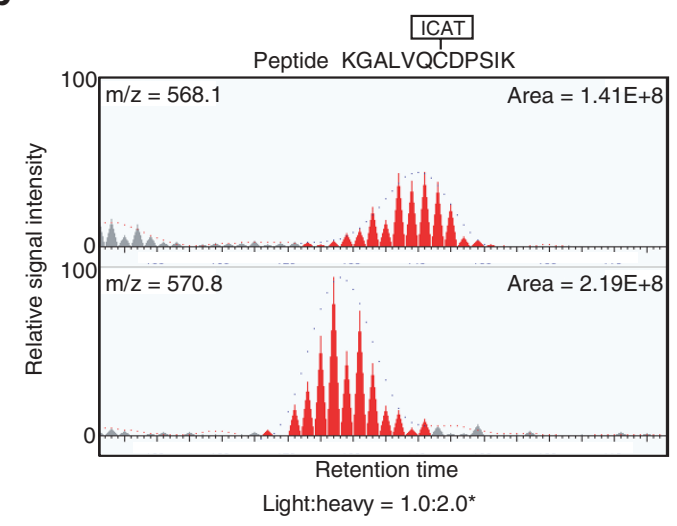

Figure 1 Identification of YDR079c-a by quantitative proteomic analysis of promoter-DNA purified Pol II PICs. (a) Schematic of the quantitative proteomic approach for the analysis of Pol II PICs. Nuclear extract from a strain carrying a temperature-sensitive allele of TBP (TBP I143N) ${ }^{27}$ was incubated with the activator Gal4-AH and a core HIS4 promoter linked to magnetic beads in the presence or absence of recombinant TATA-binding protein (rTBP). Extracts prepared from this strain are defective in formation of active transcription complexes, but addition of rTBP restores transcriptional activity ${ }^{23}$. Promoterassociated proteins were isolated. Proteins from each sample were differentially labeled with ICAT reagents and combined. After proteolysis and fractionation, peptides were analyzed by $\mu \mathrm{LC}-\mathrm{MS} / \mathrm{MS}$. In this analysis, peptides are identified and their relative abundances in the two samples are determined. This permits the distinction of core Pol II components from copurifying proteins based on the enrichment in the sample containing rTBP compared to the control sample. (b) Quantification of isotopically labeled YDR079c-a peptides. SEQUEST database searching matched the MS/MS spectrum of an ion with mass-to-charge ratio $(\mathrm{m} / \mathrm{z})$ of 568.1 to the indicated ICAT labeled peptide sequence corresponding to YDRO79c-a. Relative quantification of the isotopically heavy and normal ICAT labeled peptides was determined after reconstructing single-ion chromatograms for these peptides and summing the signal intensities for each peptide ion during the elution time with XPRESS software ${ }^{6}$. The asterisk by the light:heavy ratio indicates that the ratio was normalized.

${ }^{1}$ Institute for Systems Biology, 1441 North 34th Street, Seattle, Washington 98103-8904, USA. ${ }^{2}$ The Howard Hughes Medical Institute and Division of Basic Sciences, The Fred Hutchinson Cancer Research Center, Seattle, Washington, 98109, USA. ${ }^{3}$ Department of Chemistry, University of Washington, Seattle, Washington 98195, USA. Correspondence should be addressed to J.A.R. (jranish@systemsbiology.org). 
a

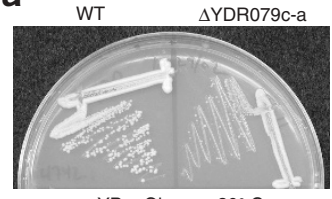

YP + Glucose $30^{\circ} \mathrm{C}$

WT

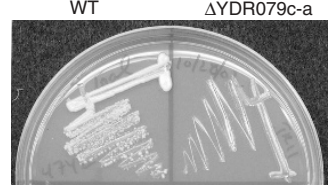

$\mathrm{YP}+$ Galactose $30^{\circ} \mathrm{C}$

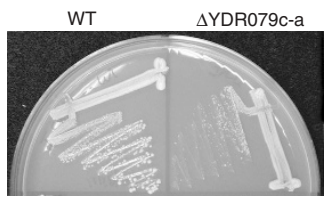

SC + Glucose - Inositol $30^{\circ} \mathrm{C}$

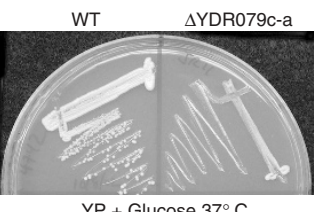

$\mathrm{YP}+$ Glucose $37^{\circ} \mathrm{C}$

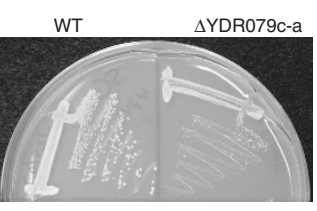

SC + Glucose $30^{\circ} \mathrm{C}$

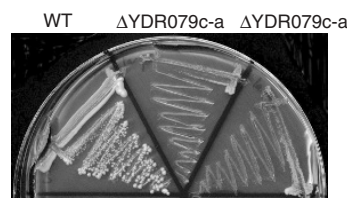

$\mathrm{SC}+$ Glycerol $30^{\circ} \mathrm{C}$ b

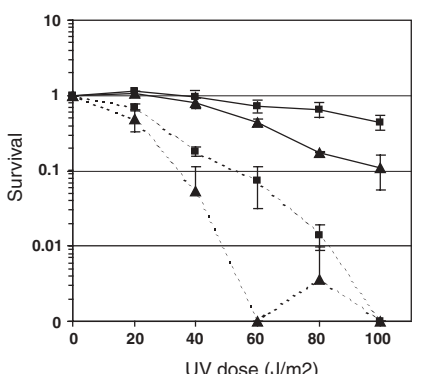

—BY4742 (WT)

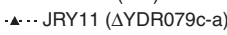

- SB207 (WT)

-.. SB260 (tfb1-101)
Figure 2 Growth phenotypes of the YDR079c-a deletion strain. (a) A YDR079c-a deletion strain $(\triangle Y$ YR079c-a) and an isogenic wild-type strain (WT) were streaked onto plates containing the indicated medium and incubated for $2-3 d$ at the indicated temperatures. YP, yeast extract/peptone medium; SC, synthetic complete medium. (b) UV sensitivity of the YDR079c-a deletion strain ( $\triangle$ YDR079c-a). Logarithmically growing YPD cultures at $30^{\circ} \mathrm{C}$ were diluted into fresh YPD, plated onto YPD plates and incubated for 30 min in the dark. Uncovered plates were irradiated with UV light (Stratalinker 2400) at the indicated doses. After incubation at $30^{\circ} \mathrm{C}$ for $2-3 \mathrm{~d}$, the surviving colonies were counted. Results are the averages from three experiments. WT, wild-type.
Among 206 proteins that were identified in a quantitative proteomics screen of RNA polymerase II (Pol II) preinitiation complexes (PICs), 49 were enriched by a factor of at least 1.9 in the specific purification ${ }^{1}$ (Fig. 1a). All the known proteins in this group are components of the Pol II transcription machinery. Two overlapping tryptic peptides from the previously uncharacterized open reading frame (ORF) YDR079c-a were enriched by a factor of $2.0 \pm 0.3(n=4)$ in the specific purification (Fig. 1 b and Supplementary Fig. 1 online). BLAST searching identified substantial similarity to potential ORFs in multiple eukaryotic organisms (Supplementary Fig. 1 online and data not shown). YDR079c-a has sequence identity of $28 \%$ and $44 \%$ similarity with human and mouse homologs. A YDR079c-a homolog was recently identified in Chlamydomonas reinhardtii as a suppressor of an ultraviolet (UV) light-sensitive mutant ${ }^{3}$. This finding is notable because the core general transcription factor IIH has a role in repairing DNA damage ${ }^{4}$.

To characterize YDR079c-a, we replaced one copy of the gene encoding it with the KanMX gene in a diploid strain. Sporulation produced four viable spores, but a slow-growth phenotype segregated 2:2 with the KanMX+ spores (six of six tetrads, data not shown). Deletion strains grew slowly on rich medium containing glucose, and the slowgrowth phenotype was more pronounced at $37^{\circ} \mathrm{C}$ (Fig. 2a). In addition, deletion strains grew very slowly on galactose, glycerol and medium lacking inositol. Thus, YDR079c-a is required for efficient growth under a variety of environmental conditions.

We next tested the YDR079c-a deletion strain for sensitivity to UV radiation. The deletion strain was sensitive to UV light (Fig. 2b), and the degree of sensitivity closely resembled that of a strain carrying a temperature-sensitive mutation in the core TFIIH subunit TFB1 (ref. 5), suggesting that YDR079c-a is required for repair of DNA damage.

To test directly whether YDR079c-a has a role in transcription initiation, we assayed basal and activated Pol II transcription activity in nuclear extracts prepared from a strain lacking YDR079c-a. Compared with extracts from a wild-type strain, extracts from the YDR079c-a deletion strain were defective in both basal and activated transcription a

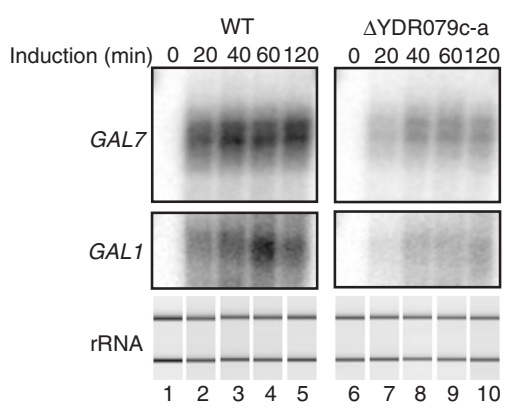

b

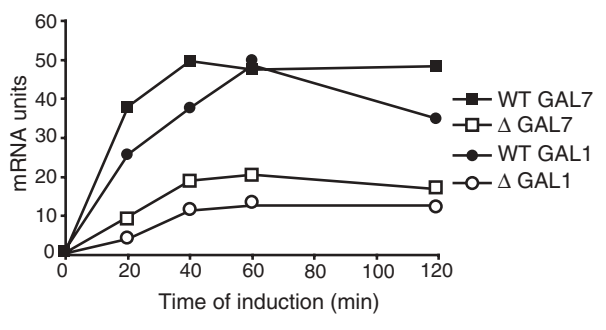

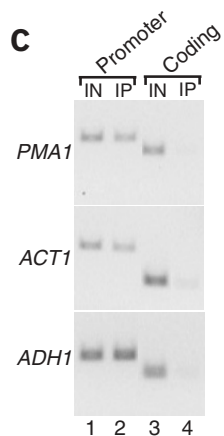

d

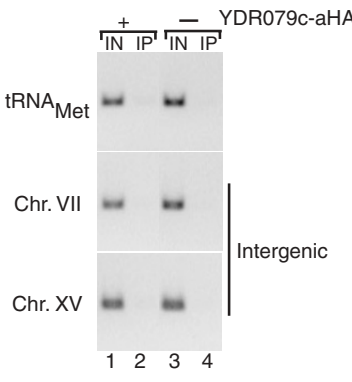

Figure 3 YDR079c-a is required for normal expression of GAL genes and is recruited to actively transcribed genes. (a) YDR079c-a is required for high-level induction of GAL7 and GAL1 by galactose. RNA was isolated from wild-type (WT) and YDR079c-a deletion ( $\triangle$ YDR079c-a) strains before or after induction with galactose for the indicated times. GAL7 and GALI mRNAs were monitored by S1 nuclease protection. Bioanalyzer 2100 (Agilent) analysis of rRNA is shown as a control for total RNA. (b) GAL7 and GAL1 mRNA levels from a were quantified. WT, wild-type strain; $\triangle$, deletion strain. (c,d) YDR079c-a is recruited to actively transcribed promoters in vivo. Chromatin immunoprecipitation analysis of YDR079c-a-HA at several genomic loci. (c) Antibodies to HA were used to immunoprecipitate cross-linked chromatin from an HA-tagged YDR079c-a strain. Immunoprecipitated DNA (IP) and input DNA (IN) were analyzed by PCR using primers to the indicated genomic loci. Products were resolved and detected on ethidium bromide-stained agarose gels. Normalized enrichment factors were 13, 9 and 7 for PMA1, ACT1 and ADH1, respectively. (d) Antibodies to HA were used to immunoprecipitate cross-linked chromatin from a HA-tagged YDR079c-a strain and an untagged strain. Immunoprecipitated DNA (IP) and input DNA (IN) were analyzed as described in c. 
assays (Supplementary Fig. 2 online; 3.0 times lower). Even though Gal4-VP16 could not overcome the transcription defect of the YDR079c-a deletion strain, it was still able to stimulate transcription in the absence of YDR079c-a (simulation by factors of 7 and 8 in deletion and wild-type strains, respectively). Thus, the defect in activated transcription reactions is probably due to the observed defect in basal transcription reactions. Notably, recombinant GST-YDR079c-a stimulated basal and activated transcription assays (by a factor of 2.0). Consistent with its proposed role as a component of Pol II PICs, YDR079c-a is required for efficient basal and activated transcription by Pol II in vitro.

To test whether YDR079c-a is required for proper gene expression in vivo, we carried out genome-wide DNA microarray analysis using poly $(\mathrm{A})^{+}$RNA from a YDR079c-a deletion strain and an isogenic wildtype strain grown in rich medium at $30^{\circ} \mathrm{C}$. Under these conditions, only three genes showed significant differences in expression levels between the two strains $(P<0.1 \%$, Supplementary Table 1 online). Levels of $M F \alpha 1$ RNA were three times lower, and levels of CUP1-1 and CUP1-2 RNA were 12 times higher and 9 times higher, respectively, in the deletion mutant than in the wild-type strain. Because the slowgrowth phenotype of the YDR079c-a deletion strain was exacerbated in response to changing environmental conditions (Fig. 2a), we reasoned that the potential role of YDR079c-a in gene expression might become evident after exposing cells to a new environmental situation. To test this idea, we assayed the kinetics of induction of GAL7 and GAL1 after exposing cells to galactose. Compared with the wild-type strain, the YDR079c-a deletion strain was defective in the extent of activation of GAL7 and GAL1 (Fig. 3a,b). These results are consistent with both the slow growth of YDR079c-a deletion strains on galactose and the in vitro transcription results showing that YDR079c-a is required for efficient transcription initiation in the presence of an activator (Supplementary Fig. 2 online). Thus, although YDR079c-a is not absolutely required for gene expression, it is required for high-level induction of GAL genes.

To test whether YDR079c-a is recruited to active promoters in vivo, we carried out chromatin immunoprecipitation experiments with a hemagglutin (HA)-tagged YDR079c-a strain. Promoter DNA from three actively transcribed genes was specifically enriched compared with DNA from downstream ORFs (Fig. 3c). We also assayed for promoter DNA from the Pol III-transcribed $\mathrm{tRNA}_{\text {Met }}$ promoter and for DNA from two intergenic regions. Compared with chromatin isolated from an untagged strain, these DNA fragments were not enriched in chromatin isolated from the YDR079c-a-HA strain (Fig. 3d).

Next, we applied the quantitative proteomics approach ${ }^{1}$ to identify proteins that potentially interact with YDR079c-a (Fig. 4a). Nuclear

\footnotetext{
Figure 4 Quantitative proteomic analysis of YDR079c-a-FLAG immunoprecipitates. (a) The quantitative proteomic approach for identifying proteins that interact with YDR079c-a. Nuclear extracts from a strain carrying epitope-tagged YDR079c-a-FLAG or wild-type YDR079c-a were used in immunoprecipitation reactions with immobilized antibodies to FLAG. Immunoprecipitated proteins from the two samples were differentially labeled with ICAT reagents and analyzed by the quantitative proteomic approach described in Figure 1. (b) Immunoprecipitates with antibody to FLAG from nuclear extracts containing epitope-tagged YDR079c-a-FLAG or wild-type YDR079c-a. 1.25\% of the immunoprecipitated samples were resolved on 4-12\% Bis-Tris gels (Invitrogen) and stained with silver. (c) Summary of results from the quantitative proteomic analysis of YDR079c-a-FLAG immunoprecipitates. The most significantly enriched proteins are listed $(P<0.01)$. The number of unique peptide ions identified from the protein is given in parentheses after the protein symbol. The number of peptide pairs quantified followed by the number of unique peptide sequences used for quantification is given in parentheses after the abundance ratio.
}

extracts from a strain carrying epitope-tagged YDR079c-a-FLAG or wild-type YDR079c-a were used in immunoprecipitation reactions with antibodies to FLAG. Analysis of an aliquot of the eluted proteins by SDS-PAGE and silver staining showed the complexity of the samples (Fig. 4b). The remaining samples were differentially labeled with ICAT reagents and prepared for quantitative mass spectrometry as described ${ }^{1,6}$. Proteins that potentially interact with YDR079c-a are identified by the relative enrichment of their peptides in the YDR079ca-FLAG immunoprecipitation compared with the wild-type immunoprecipitation. Of the 93 quantified proteins, 82 (88\%) were not significantly enriched $(P>0.01 \text {, Supplementary Table } 2 \text { online })^{7}$. Three core components of general transcription and DNA repair factor IIH, TFB1, TFB2 and TFB4, were the most significantly enriched proteins in the YDR079c-a-FLAG immunoprecipitation (enriched by a factor of $\geq 10.5, P \leq 1 \times 10^{-23}$; Fig. $4 c$ ). In addition, four other core TFIIH subunits were enriched by factors of 1.9-3.5 in the specific immunoprecipitation $(P<0.01)$. KIN28 and CCL1, components of the kinase subcomplex TFIIK of TFIIH, were not detected. In addition to the TFIIH subunits, ribosomal subunits (RPL18A or B and RPS23A or B), a snoRNA binding protein (UTP8) and a metabolic enzyme (LEU1) were enriched by a factor of more than $2(P<0.01)$.

To test whether YDR079c-a is a component of TFIIH, we monitored levels of YDR079c-a during the purification of TFIIH. The core TFIIH subunit TFB4 was immunoprecipitated from a whole-cell extract derived from a strain carrying epitope-tagged TFB4-FLAG and YDR079c-a-HA. Immunoblotting showed that, whereas most YDR079c-a-HA and core TFIIH subunit TFB1 coprecipitated with TFB4-FLAG, TFIIK subunits TFB3 and KIN28, and PIC components $\mathrm{TBP}$ and RBP3, were not detectable in the precipitates using antibody to FLAG (Fig. 5a). Unlike the YDR079c-a immunoprecipitation, TFB3 was not detectably enriched in the TFB4 immunoprecipitation. This discrepancy is due to different wash conditions used in the two experiments (data not shown). The complexity and apparent fragile nature

a

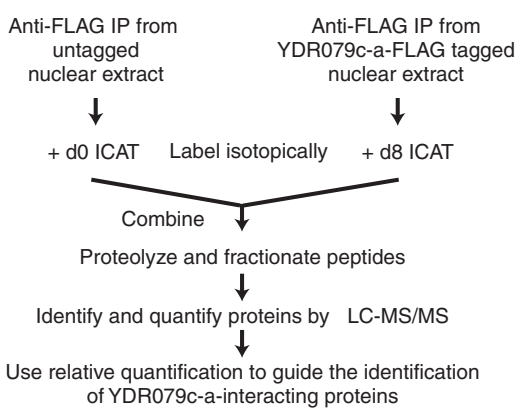

C

\begin{tabular}{|l|c|c|c|l|}
\hline \multicolumn{1}{|c|}{ Protein } & Abundance ratio & S.d. & P value & \multicolumn{1}{|c|}{ Function } \\
\hline TFB2 (3) & $\geq 16.2(4 / 2)$ & & $5.32 \mathrm{E}-31$ & TFIIH 55 kDa subunit \\
TFB4 (3) & $\geq 11.8(4 / 2)$ & & $9.42 \mathrm{E}-25$ & TFIIH 37 kDa subunit \\
TFB1 (4) & $\geq 11.2(4 / 2)$ & & $1.01 \mathrm{E}-23$ & TFIIH 75 kDa subunit \\
TFB3 (2) & $\geq 3.5(3 / 2)$ & & $1.69 \mathrm{E}-07$ & TFIIH 38 kDa subunit \\
RPL18A or B (3) & $4.4(4 / 1)$ & 0.8 & $6.12 \mathrm{E}-07$ & Ribosomal protein L18B \\
RPS23A or B (2) & $2.9(2 / 1)$ & 0.3 & $3.99 \mathrm{E}-05$ & Ribosomal protein S23A \\
RAD3 (2) & $\geq 2.6(2 / 2)$ & & $9.55 \mathrm{E}-05$ & TFIIH subunit \\
SSL2 (4) & $\geq 2.6(6 / 3)$ & & $9.55 \mathrm{E}-05$ & TFIIH helicase \\
UTP8 (1) & $\geq 2.3(1 / 1)$ & & $5.66 \mathrm{E}-04$ & snoRNA binding activity \\
LEU1 (11) & $\geq 2.2(6 / 4)$ & & $1.37 \mathrm{E}-03$ & 3-isopropylmalate dehydratase \\
SSL1 (2) & $1.9(2 / 2)$ & 0.1 & $6.21 \mathrm{E}-03$ & TFIIH subunit \\
\hline
\end{tabular}

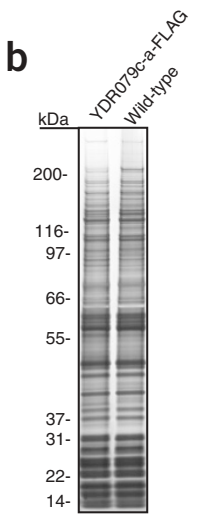


of yeast TFIIH is well documented ${ }^{8-10}$. But a five-to-seven-subunit, core TFIIH complex that interacts with the two-to-three-subunit TFIIK complex has been described in yeast and humans ${ }^{9,11,12}$.

We separated complexes containing TFB4 from the immunoprecipitation with antibody to FLAG by MonoQ chromatography. TFB4 eluted with TFB1 in three distinct peaks at $0.25 \mathrm{M}, 0.32 \mathrm{M}$ and $0.67 \mathrm{M}$ $\mathrm{KCl}$ (Fig. 5b). YDR079c-a cofractionated with the TFB4-TFB1 peaks eluting at $0.32 \mathrm{M}$ and $0.67 \mathrm{M} \mathrm{KCl}$, with most YDR079c-a in the former peak. These two peaks contain most of the TFB4 and TFB1.

We further analyzed MonoQ fractions by SDS-PAGE and silver staining. Fraction 36 contained seven proteins that cochromatographed with the main peak of YDR079c-a, TFB1 and TFB4 (Fig. 5b,c). In a parallel purification from a strain carrying FLAGtagged TFB4 and wild-type YDR079c-a, we excised bands with identical mobilities to those indicated in Figure $\mathbf{5 c}$ from the gel, digested them with trypsin and analyzed them by mass spectrometry. Sequence database searching identified peptides from the core TFIIH subunits TFB1, TFB2, SSL1 and TFB4 (Fig. 5d). We also identified four peptides from YDR079c-a in a band that migrated at $6 \mathrm{kDa}$. The doublet at $60 \mathrm{kDa}$ was not observed in the parallel purification. Because LEU1 and SSA1, SSA2 and SSB1 or SSB2 did not exactly comigrate with the core TFIIH subunits, they are probably contaminants. The absence of RAD3 and SSL2 in this preparation of TFIIH is probably explained by the propensity of these subunits to dissociate from TFIIH complexes during MonoQ chromatography ${ }^{9,10}$. It is possible that these subunits copurified with the $0.67 \mathrm{M}$ peak (Fig. 5b).

To probe the mechanism of YDR079c-a action, we monitored the ability of extract from a YDR079c-a deletion strain to form PICs using an immobilized template assay. Compared with the wild-type strain, the YDR079c-a deletion strain showed no defect in the stable recruitment of PIC components, except for TFIIH components TFB1 and KIN28 (Fig. 6a). Furthermore, addition of glutathione S-transferase (GST)-tagged YDR079c-a specifically stimulated recruitment of TFIIH components. Notably, some TFIIH was recruited to promoters in the absence of YDR079c-a (Fig. 6a), and these PICs were active (Supplementary Fig. 2 online). Thus, YDR079c-a is required for stable recruitment of TFIIH to promoters, and taken together with the previous findings, these results show that YDR079c-a is a new core TFIIH subunit, which we named TFB5.

Although the role of TFB5 in DNA-damage repair seems to be conserved throughout evolution, there are a number of differences in TFB5 function between yeast and humans. In humans, TFB5 affects the steady-state levels of TFIIH because cells carrying mutations in TFB5 have decreased levels of TFIIH components, and transient overexpression of TFB5 increases levels of TFIIH components ${ }^{2,13}$. In contrast, deletion of TFB5 in yeast does not affect levels of TFIIH subunits (Fig. 6b). The ability of recombinant TFB5 to stimulate transcription activity (Supplementary Fig. 2 online) and TFIIH

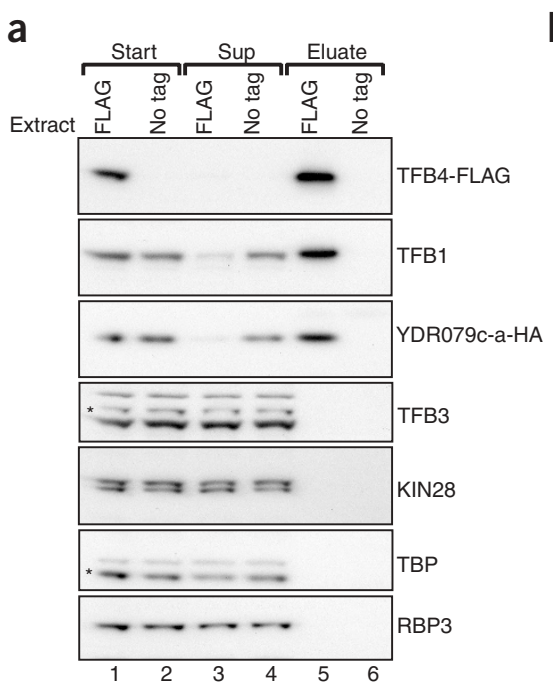

b
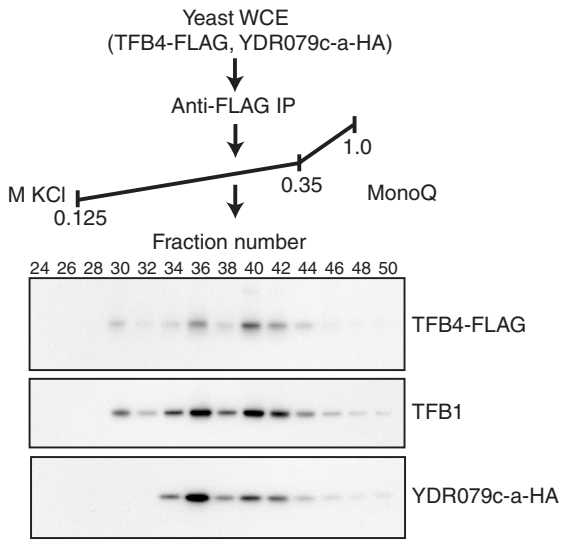

C

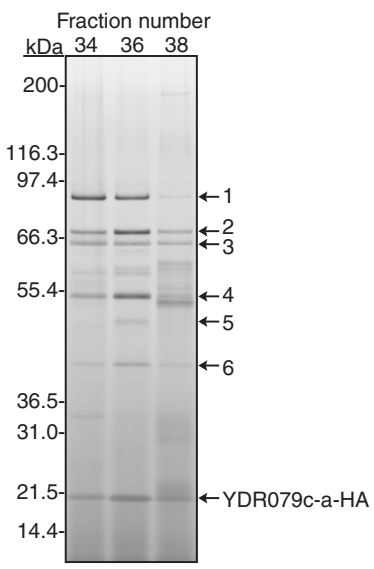

d

\begin{tabular}{|clc|}
\hline Band & ORF(s) & Number of peptides \\
\hline 1 & LEU1 & 32 \\
2 & TFB1 & 25 \\
3 & SSA1 & 25 \\
& SSA2 & 7 \\
& SSB1 or 2 & 15 \\
& TFB1 & 7 \\
4 & TFB2 & 21 \\
& AR09 & 2 \\
5 & SSL1 & 17 \\
& YHR111W & 1 \\
6 & TFB4 & 14 \\
& ACT1 & 3 \\
& FBA1 & 2 \\
7 & YDR079c-a & 4 \\
\hline
\end{tabular}

Figure 5 YDRO79c-a is a subunit of core TFIIH. (a) YDR079c-a coimmunoprecipitated with TFB4. Whole-cell extracts from strains containing HA-tagged YDR079c-a with or without FLAG-tagged TFB4 were used in immunoprecipitation reactions with FLAG-M2 agarose beads. Whole-cell extracts (Start), supernatants (Sup) and eluates were analyzed by SDS-PAGE followed by immunoblotting. $0.75 \%$ of the whole-cell extracts and supernatants and $17 \%$ of the eluates were analyzed. Asterisks indicate the bands corresponding to the protein that was probed for. (b) YDR079c-a copurified with affinity-purified TFB4-TFB1 complexes on MonoQ. Whole-cell extracts (WCE) from the strain containing TFB4-FLAG and YDR079c-a-HA was used to immunoprecipitate TFB4-FLAG as described in a. Immunopurified complexes containing TFB4 were separated on a MonoQ column. $10 \mu$ l of the indicated fractions were analyzed by SDS-PAGE followed by immunoblotting. (c) Silver-stained SDS-PAGE analysis of the peak YDR079c-a MonoQ fractions. $40 \mu$ l of the indicated fractions were precipitated with trichloroacetic acid and analyzed by SDS-PAGE followed by silver staining. Numbers on the right indicate the position of the bands that were analyzed by $\mu \mathrm{LC}-\mathrm{MS} / \mathrm{MS}$. The identity of YDR079c-a-HA is inferred from its mobility and elution profile in the immunoblot from b. (d) $\mu \mathrm{LC}-\mathrm{MS} / \mathrm{MS}$ analysis of a core TFIIH complex identifies YDR079c-a. Numbered bands in $\mathbf{c}$ were excised from a gel of the peak YDR079c-a fraction from a parallel purification of TFB4 from a strain containing TFB4-FLAG and wild-type YDR079c-a. Proteins were digested with trypsin and analyzed by $\mu \mathrm{LC}-\mathrm{MS} / \mathrm{MS}$. The table lists the ORFs and the number of peptides that were identified from each gel slice. 
recruitment (Fig. 6a) is consistent with this finding. Despite this difference, it is possible that lack of TFB5 affects the stability or structure of the TFIIH complex in yeast. Furthermore, in humans the absence of TFB5 does not seem to affect transcription initiation in vitro ${ }^{14}$.

Our results have three main implications. First, the identification of TFB5 probably completes the description of TFIIH composition. Second, this study shows the usefulness of mass spectrometry-based quantitative proteomics for the discovery and subsequent characterization of new components of protein complexes. Third, before this study, despite a clear association between the DNA-damage repair defect in the TTD-A form of the human tricothiodystrophy disorder and TFIIH, the gene responsible for the disorder had not been identified ${ }^{13}$. The identification of TFB5 as a core TFIIH component prompted an investigation of whether human TFB5 is part of mammalian TFIIH and, by extension, whether it is involved in TTD- $\mathrm{A}^{2}$. This led to the rapid elucidation of the molecular defect underlying TTD- $\mathrm{A}^{2}$.

\section{METHODS}

Plasmids. The pYM series plasmids and pUG6 have been described ${ }^{15,16}$. We amplified the triple FLAG tag by PCR from p3FLAG-KanMX ${ }^{17}$ using primers FLAG1 and FLAG5. We cloned the fragment digested by BamHI and Bss HI into pYM1 digested by BamHI and BssHI to create pJR200. We amplified the YDR079c-a ORF by PCR from Saccharomyces cerevisiae genomic DNA with BamHI and NotI restriction sites at the $5^{\prime}$ and $3^{\prime}$ ends, respectively, using primers Bam079 and Not079. We digested the fragment and cloned it into pGEX6P-1 (Amersham) digested by BamHI and NotI to create the GST-YDR079c-a expression construct, pJR100. Primer sequences are available on request.

Yeast methods. Yeast strains used in this study are listed in Supplementary Table 3 online. We replaced one copy of the gene encoding YDR079c-a (TFB5) with KanMX using a PCR-mediated strategy in diploid strain BY4743 (ref. 16). After sporulating KanMX+ strains, we dissected tetrads and verified the replacement of TFB5 with KanMX by PCR. We epitope-tagged TFB5 at its $3^{\prime}$ end using the following PCR-based strategies. We created JRY14 containing triply FLAG-tagged TFB5 using pJR200 and BY4742. We created JRY191 and JRY193 containing six HA-tagged TFB5 using pYM3 and SHY365 and BJ5460, respectively. We created SHY365 containing triply FLAG-tagged TFB4 using p3FLAG-KanMX and BJ5460.

RNA analysis. For the DNA microarray analysis, we grew strains BY4742 and JRY11 in YPD ( $1 \%$ yeast extract, $2 \%$ peptone, $2 \%$ glucose) at $30^{\circ} \mathrm{C}$ to $\mathrm{A}_{600}$ of 1.5 . We removed $100-\mathrm{ml}$ aliquots, pelleted cells at $3,000 \mathrm{~g}$ for $5 \mathrm{~min}$, removed the supernatants and froze the cells on dry ice. We isolated total RNA by hot acid phenol extraction ${ }^{18}$ and quantified it by UV absorbance at $260 \mathrm{~nm}$ and by analysis on a 2100 Bioanalyzer (Agilent). We isolated poly $(\mathrm{A})^{+}$RNA from total RNA and generated cDNAs labeled with Cy3 or Cy5 dUTP by reverse transcription as described ${ }^{19}$. We combined cDNAs from BY4742 and JRY11, labeled with Cy3 and Cy5, respectively, and hybridized them to yeast whole-genome oligonucleotide microarrays. We carried out a dye-flip experiment to control for differences in labeling efficiency. Hybridization was done for $16 \mathrm{~h}$ at $37^{\circ} \mathrm{C}$ in DIG Easy Hybe (Roche) containing $0.5 \mathrm{mg} \mathrm{ml}^{-1}$ of yeast tRNA and $100 \mu \mathrm{g} \mathrm{ml}^{-1}$ of salmon sperm DNA. We washed microarray slides as described ${ }^{19}$ and scanned them using a ScanArray 5000 microarray analyzer (Packard BioScience). We processed images using the microarray spot finding and quantification software AnalyzerDG. We located fluorescent spots and, for each dye background, estimated the intensity and subtracted it from the mean intensity in each spot area. We then normalized the values so that the medians of all Cy 3 and all Cy5 intensities were equal ${ }^{19,20}$. We used a previously established statistical method to assign the maximum likelihood of differential expression for each gene ${ }^{19}$. We estimated the false-positive rate by carrying out an additional microarray experiment in which equal amounts of Cy3- and Cy5-labeled cDNA from strain BY4742 were hybridized against themselves. The eighth highest likelihood score in the control experiment was chosen to establish a threshold of $\sim 0.1 \%$ false positives in the real experiment.

For the galactose induction time course experiment, we grew strains BY4742 and JRY11 in YPR ( $1 \%$ yeast extract, $2 \%$ peptone, $2 \%$ raffinose) at $30^{\circ} \mathrm{C}$ to an a

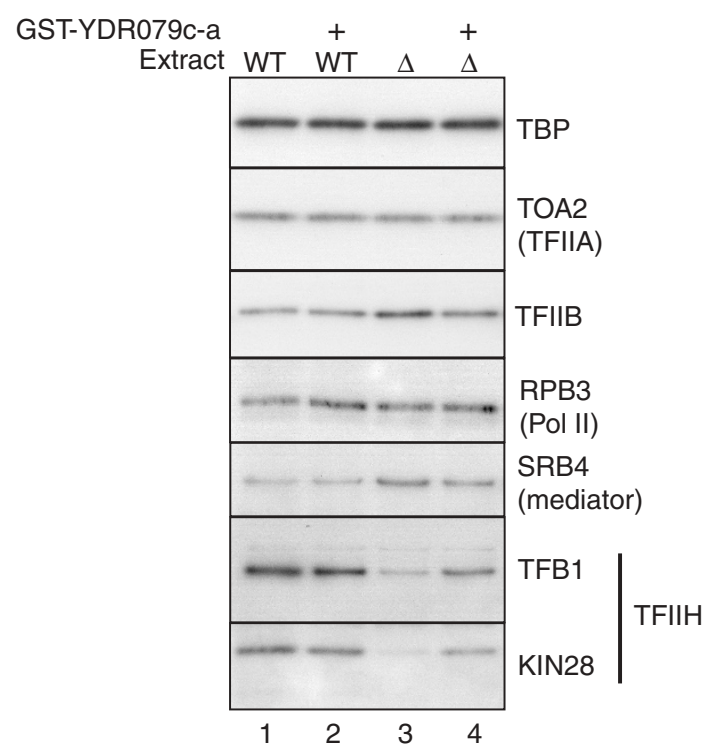

b

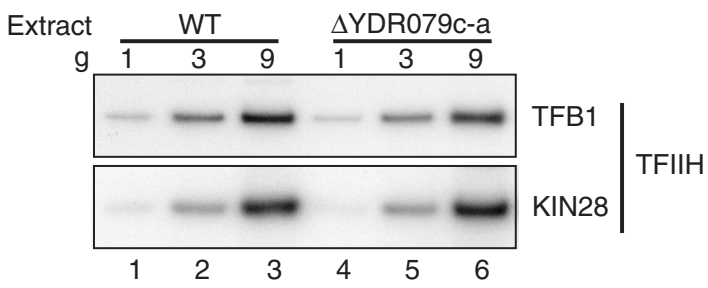

Figure 6 YDR079c-a is required for stable recruitment of TFIIH to a promoter but not for stability of TFIIH subunits. (a) Immobilized promoter assay with a YDR079c-a deletion extract. Nuclear extracts from a wild-type strain (WT) and a YDR079c-a deletion strain $(\Delta)$ were incubated with an immobilized HIS4 promoter template for 40 min. GST-YDR079c-a was included in the indicated reactions. Templates were washed, and promoterbound proteins were eluted and analyzed by SDS-PAGE followed by immunoblotting with antibodies to the indicated proteins. (b) Stability of TFIIH subunits in the absence of YDR079c-a. Increasing amounts of nuclear extract from the indicated strains (WT, wild-type; $\triangle$ YDR079c-a, deletion) were resolved by SDS-PAGE and analyzed by immunoblotting with antibodies to TFB1 and KIN28.

$\mathrm{A}_{600}$ of 1.0. We removed 50-ml aliquots for the zero time points and collected cells as described above. We added galactose to a final concentration of $2 \%$ to the remaining cultures and removed 50-ml samples at the indicated time points. We processed cells and isolated total RNA as described above. We carried out S1 nuclease protection assays essentially as described ${ }^{21}$ with $20 \mu \mathrm{g}$ of RNA. We resolved reaction products on $8 \%$ denaturing polyacrylamide gels, analyzed the dried gels using a PhosphorImager (Molecular Dynamics) and quantified them using IQMACv2.1 software.

Chromatin immunoprecipitation. We grew $50 \mathrm{ml}$ of yeast strain JRY193 (TFB5-HA) and the isogenic wild-type strain BJ5460 to $\mathrm{A}_{600}$ of $\sim 1.5$ in YPD at $30{ }^{\circ} \mathrm{C}$. We added formaldehyde to a final concentration of $1 \%$ for $15 \mathrm{~min}$. We quenched cross-linking by adding glycine to a final concentration of $125 \mathrm{mM}$. We collected cells by centrifugation, washed them successively with phosphatebuffered saline containing $125 \mathrm{mM}$ of glycine and then with phosphate-buffered saline alone and resuspended them in $400 \mu \mathrm{l}$ of chromatin immunoprecipitation lysis buffer (CLB; 50 mM HEPES buffer (pH 7.5), $140 \mathrm{mM} \mathrm{NaCl}, 1 \%$ Triton X$100,0.1 \%$ sodium deoxycholate and $1 \mathrm{mM}$ EDTA) containing protease 
inhibitors. After adding $\sim 400 \mu \mathrm{l}$ of glass beads, we disrupted cells by vortexing for $1 \mathrm{~h}$ at $4{ }^{\circ} \mathrm{C}$ and collected the supernatant. We sheared chromatin by sonicating to yield an average DNA fragment size of $450 \mathrm{bp}$. We centrifuged the chromatin solution twice at 14,000 r.p.m. for $5 \mathrm{~min}$, isolated the supernatant and determined protein concentration using a dye-binding assay (BioRad).

We diluted cross-linked chromatin ( 1 mg of protein) to $5 \mathrm{mg} \mathrm{ml}^{-1}$ in CLB and set aside $1 \%$ of this solution for the input controls. We incubated chromatin with antibodies to HA (Covance Research Products) overnight at $4{ }^{\circ} \mathrm{C}$. We precipitated immune complexes with Protein G Sepharose (Amersham) and washed them twice with $1 \mathrm{ml}$ of CLB, twice with $1 \mathrm{ml}$ of CLB containing $500 \mathrm{mM}$ of NaCl , twice with $1 \mathrm{ml}$ of wash buffer ( $10 \mathrm{mM}$ Tris (pH 8.0), $250 \mathrm{mM}$ $\mathrm{LiCl}, 0.5 \%$ Nonidet P-40, 0.5\% sodium deoxycholate and $1 \mathrm{mM}$ EDTA) and twice with $1 \mathrm{ml}$ of TE 8 . We eluted chromatin by incubating beads two times in $85 \mu$ of elution buffer ( $50 \mathrm{mM}$ Tris buffer ( $\mathrm{pH} 8.0$ ), $1 \%$ SDS and $10 \mathrm{mM}$ EDTA) for $10 \mathrm{~min}$ at $65^{\circ} \mathrm{C}$. We reversed crosslinks by incubating the eluted samples and the input controls at $65^{\circ} \mathrm{C}$ overnight. We purified DNA with a Qiagen PCR purification kit.

To amplify signals, we carried out PCR reactions $(25 \mu \mathrm{l})$ with the appropriate primers $(1 \mu \mathrm{M})$ and $1.0 \mathrm{U}$ Taq. We used $1-2 \%$ of the immunoprecipitated DNA and $4 \%$ of the input DNA as templates for PCR. After 2 min at $95^{\circ} \mathrm{C}$, reactions were cycled 25 times at $95^{\circ} \mathrm{C}$ for $0.5 \mathrm{~min}, 50{ }^{\circ} \mathrm{C}$ for $0.5 \mathrm{~min}$ and $72{ }^{\circ} \mathrm{C}$ for $0.75 \mathrm{~min}$, followed by a 7 -min incubation at $72^{\circ} \mathrm{C}$. We resolved products on $2 \%$ agarose gels containing ethidium bromide and visualized them by exposure to UV light. We quantified products by densitometry using IQMACv1.2 software. The fraction of immunoprecipitated material for a specific fragment was calculated by dividing the amount of PCR product obtained with immunoprecipitated DNA by the amount obtained with input DNA. We used these values to calculate the relative enrichment of promoter DNA to coding DNA. The location of the PCR products with respect to the translation start site were $-388 /-51$ PMA1 promoter, +2018/+2290 PMAl coding, -388/-46 ACT1 promoter, +1223/+1427 ACT1 coding, -235/-13 ADH1 promoter, +884/+1013 ADH1 coding and $-292 /-11$ tRNA $_{\text {Met }}$.

Preparation of extracts. We prepared nuclear extracts as described ${ }^{22}$. We prepared whole-cell extracts from $2-31$ of cells grown to an $\mathrm{A}_{600}$ of $\sim 3.0$ at $30^{\circ} \mathrm{C}$. We pelleted the cells, washed them in $50 \mathrm{ml}$ of lysis buffer $(50 \mathrm{mM}$ Tris buffer (pH 7.8), $150 \mathrm{mM} \mathrm{KCl}, 1 \mathrm{mM} \mathrm{EDTA}$ and 20\% glycerol) per liter of cells and resuspended in $5 \mathrm{ml}$ of lysis buffer per liter of cells containing $1 \mathrm{mM}$ dithiothreitol and protease inhibitors ( $1 \mathrm{mM}$ phenylmethylsulfonyl fluoride, $2 \mathrm{mM}$ benzamidine, $0.6 \mu \mathrm{M}$ leupeptin, $2 \mu \mathrm{M}$ pepstatin A and $3 \mu \mathrm{M}$ chymostatin). After disrupting cells by passage through a microfluidizer (Microfluidics), we clarified extracts by centrifugation at $10,000 \mathrm{~g}$ for $10 \mathrm{~min}$ and then at $100,000 \mathrm{~g}$ for $60 \mathrm{~min}$. Protein concentrations were determined using the Bio-Rad dye-binding assay.

Protein purification. rTBP, Gal4-AH and Gal4-VP16 have been described ${ }^{23}$. We grew a 50-ml culture of Escherichia coli strain BL21 transformed with pJR100 to an $\mathrm{A}_{600}$ of $\sim 1.0$ and induced expression of GST-TFB5 with $1 \mathrm{mM}$ of IPTG for $2 \mathrm{~h}$ at $37^{\circ} \mathrm{C}$. We collected cells, washed them in $50 \mathrm{ml}$ of $20 \mathrm{mM}$ Tris buffer ( $\mathrm{pH} 8.0$ ) and disrupted them by adding Bugbuster (Novagen, $5 \mathrm{ml}$ per $\mathrm{g}$ of cells) containing protease inhibitors and Benzonase (Novagen; $1 \mu \mathrm{l}$ per $\mathrm{ml}$ Bugbuster). After $20 \mathrm{~min}$, we centrifuged the extract at 16,000 $\mathrm{g}$ for $20 \mathrm{~min}$. We passed the clarified extract over $0.2 \mathrm{ml}$ of Glutathione Sepharose 4B (Amersham). After washing the resin, we eluted GST-TFB5 with $10 \mathrm{mM}$ of glutathione according to the manufacturer's suggestions. Protein was dialyzed against $20 \mathrm{mM}$ of HEPES buffer ( $\mathrm{pH}$ 7.9), $100 \mathrm{mM}$ potassium acetate, $1 \mathrm{mM}$ EDTA, 10\% glycerol and $1 \mathrm{mM}$ dithiothreitol. The protein was estimated to be $>99 \%$ pure by analysis on Coomassie-stained SDS-PAGE.

For the quantitative proteomic analysis of YDR079c-a-FLAG immunoprecipitates, we diluted $12.5 \mathrm{mg}$ of nuclear extract from JRY14 (TFB5-FLAG) and BWG1-7a strains to $5 \mathrm{ml}$ in Buffer A (20 mM HEPES buffer (pH 7.6), $100 \mathrm{mM}$ potassium acetate, $1 \mathrm{mM}$ EDTA, $5 \mathrm{mM}$ magnesium acetate, $0.05 \%$ Nonidet $\mathrm{P}$ 40 and protease inhibitors), incubated it at $22{ }^{\circ} \mathrm{C}$ for $10 \mathrm{~min}$ and then centrifuged it at $3,000 \mathrm{~g}$ for $2 \mathrm{~min}$. We prepared FLAG-M2 agarose beads (Sigma) by washing with $20 \mathrm{ml}$ of Buffer A, followed by incubation in $4 \mathrm{ml}$ of $0.1 \mathrm{M}$ glycine ( $\mathrm{pH}$ 3.5) for $15 \mathrm{~min}$ and equilibration in $10 \mathrm{ml}$ of Buffer A. We incubated the clarified extracts with $0.5 \mathrm{ml}$ of FLAG-M2 agarose beads for $2 \mathrm{~h}$ at $4{ }^{\circ} \mathrm{C}$ and then washed beads twice with $12 \mathrm{ml}$ of Buffer A and then twice with
$12 \mathrm{ml}$ of Buffer B (Buffer A containing $0.0025 \%$ Nonidet P-40 and no protease inhibitors). We eluted proteins by incubating beads in $0.5 \mathrm{ml}$ of Buffer $\mathrm{B}$ containing $0.1 \mathrm{mg} \mathrm{ml}^{-1}$ triple FLAG-epitope peptide (Sigma) two times for $30 \mathrm{~min}$ each at $22{ }^{\circ} \mathrm{C}$. Proteins were concentrated and buffer was exchanged to $20 \mathrm{mM}$ Tris buffer (pH 8.3), $1 \mathrm{mM}$ EDTA and $75 \mathrm{mM} \mathrm{NaCl}$ in Microcon 10 devices (Amicon). Final volumes were $25 \mu \mathrm{l}$ and protein concentrations were estimated to be $1.6 \mathrm{mg} \mathrm{ml}^{-1}$ by silver-stained SDS-PAGE.

We carried out the small-scale TFB4-FLAG immunoprecipitation studies as described for the YDR079c-a-FLAG immunoprecipitation with the following modifications. We diluted $1 \mathrm{mg}$ of whole-cell extract from JRY191 (TFB4FLAG, YDR079c-a-HA) and JRY193 (YDR079c-a-HA) strains to $2.5 \mathrm{mg} \mathrm{ml}^{-1}$ in lysis buffer containing $250 \mathrm{mM} \mathrm{KCl}, 0.05 \%$ Nonidet P-40 and protease inhibitors. We also prepared FLAG-M2 agarose beads in this lysis buffer. After incubating extracts with $14 \mu \mathrm{l}$ of beads, we washed the beads four times with $1 \mathrm{ml}$ of lysis buffer containing $300 \mathrm{mM} \mathrm{KCl}, 0.05 \%$ Nonidet P- 40 and protease inhibitors, and eluted proteins with three $20-\mu l$ incubations in lysis buffer containing $150 \mathrm{mM} \mathrm{KCl}, 0.0025 \%$ Nonidet P-40 and $0.1 \mathrm{mg} \mathrm{ml}^{-1}$ triple FLAG-epitope peptide for $15 \mathrm{~min}$ each at $22{ }^{\circ} \mathrm{C}$. We precipitated proteins with TCA, resuspended them in LDS sample loading buffer, heated them to $80{ }^{\circ} \mathrm{C}$ for $10 \mathrm{~min}$, resolved them on $4-12 \%$ Bis-Tris gels (Invitrogen) and transferred them to Immobilon membranes. We probed blots with the indicated antibodies, followed by secondary antibodies to mouse or rabbit conjugated to horseradish peroxidase. Proteins were detected by ECL (Amersham).

We generated the starting material for the MonoQ chromatographic analysis of TFB4-FLAG complexes by immunoprecipitating TFB4-FLAG from $320 \mathrm{mg}$ of whole-cell extract from JRY191 strain with $3.75 \mathrm{ml}$ of FLAG-M2 agarose beads as described above. We eluted proteins with three $3.1-\mathrm{ml}$ incubations in lysis buffer containing $150 \mathrm{mM} \mathrm{KCl}, 0.0025 \%$ Nonidet P-40 and $0.1 \mathrm{mg} \mathrm{ml}^{-1}$ triple FLAG-epitope peptide for $15 \mathrm{~min}$ each at $22^{\circ} \mathrm{C}$. After concentrating the eluate to $1 \mathrm{ml}$ in a Centriprep 10 device (Amicon), we adjusted the $\mathrm{KCl}$ concentration to $0.125 \mathrm{M}$ by diluting the sample with lysis buffer containing $1 \mathrm{mM}$ of dithiothreitol and protease inhibitors. We loaded the sample onto a MonoQ HR5/5 column equilibrated in lysis buffer containing $0.125 \mathrm{M} \mathrm{KCl}, 1 \mathrm{mM}$ dithiothreitol and protease inhibitors at $0.5 \mathrm{ml} \mathrm{min}^{-1}$. We ran a $20-\mathrm{ml}$ gradient to $0.35 \mathrm{M} \mathrm{KCl}$, followed by a gradient to $1 \mathrm{M} \mathrm{KCl}$ in $5 \mathrm{ml}$. We collected $1-\mathrm{ml}$ fractions. Aliquots were analyzed by SDS-PAGE as described above.

Immobilized promoter assays. We carried out immobilized promoter assays as described $^{1,23}$. For the experiment in Figure 6, we incubated clarified nuclear extracts $\left(\sim 3.6 \mathrm{mg} \mathrm{ml}^{-1}\right)$ with immobilized templates $(\sim 500 \mathrm{fmol})$ in $50-100 \mu \mathrm{l}$ of transcription buffer (10 mM HEPES buffer ( $\mathrm{pH} 7.6), 100 \mathrm{mM}$ potassium glutamate, $10 \mathrm{mM}$ magnesium acetate, $5 \mathrm{~mm}$ EGTA and 3.5\% glycerol) with 250 ng of HaelII-digested E. coli DNA for $40 \mathrm{~min}$ at $22^{\circ} \mathrm{C}$. We added GSTYDR079c-a (100 ng) during PIC formation where indicated. The templates were concentrated with a magnet and washed three times with $1 \mathrm{ml}$ of transcription buffer containing $0.1 \%$ Nonidet P-40 and $2.5 \mathrm{mM}$ dithiothreitol. We resuspended templates in $10 \mu \mathrm{l}$ of Pst $\mathrm{I}$ buffer $(100 \mathrm{mM} \mathrm{NaCl}, 50 \mathrm{mM}$ Tris- $\mathrm{HCl}$ ( $\mathrm{pH} 7.9$ ) and $10 \mathrm{mM} \mathrm{MgCl}_{2}$ ) with $10 \mathrm{U}$ Pst I for $30 \mathrm{~min}$ at $37^{\circ} \mathrm{C}$, concentrated them with a magnet and recovered the supernatants. Samples were analyzed by SDS-PAGE and immunoblotting as described above.

In vitro transcription. We carried out in vitro transcription reactions as described $^{23,24}$. We incubated $90 \mu \mathrm{g}$ of clarified nuclear extract with $0.15 \mu \mathrm{g}$ of HIS4 transcription template pSH515 in $25 \mu \mathrm{l}$ of transcription buffer containing $2.5 \mathrm{mM}$ dithiothreitol, $10 \mathrm{U}$ ribonuclease inhibitor (Promega), $200 \mu \mathrm{g}$ phosphocreatine and $200 \mathrm{ng}$ phosphocreatine kinase for $40 \mathrm{~min}$ at $22{ }^{\circ} \mathrm{C}$. We included Gal4-VP16 (25 ng) and GST-YDR079c-a (25 ng) in the incubation where indicated. We initiated transcription by adding each nucleotide triphosphate to $0.4 \mathrm{mM}$. Reactions were stopped after $40 \mathrm{~min}$ by adding $180 \mu \mathrm{l}$ of stop solution $\left(0.1 \mathrm{M}\right.$ sodium acetate, $10 \mathrm{mM}$ EDTA, $0.5 \%$ SDS and $15 \mu \mathrm{g} \mathrm{ml}^{-1}$ tRNA). We extracted RNA with phenol-chloroform, precipitated it with ethanol and analyzed it by primer extension ${ }^{24}$. The assay detects transcripts that are at least $\sim 100$ nucleotides in length. Products were quantified by Phosphorimager (Molecular Dynamics).

Isotopic labeling and quantitative mass spectrometry. We isotopically labeled proteins with heavy and normal versions of ICAT and carried out pro- 
teolysis as described ${ }^{1}$. We diluted the labeled peptides with an equal volume of Buffer C (5 mM KH${ }_{2} \mathrm{PO}_{4}$ (pH 3.0) and $25 \%$ acetonitrile) and adjusted the $\mathrm{pH}$ to 3 with $10 \%$ trifluoroacetic acid. We loaded peptides onto SCX cartridges (ABI) and successively eluted them with $0.75 \mathrm{ml}$ of Buffer $\mathrm{C}$ containing $40 \mathrm{mM}, 200 \mathrm{mM}, 350 \mathrm{mM}$ and $600 \mathrm{mM} \mathrm{KCl}$. We purified labeled peptides over monomeric avidin cartridges (ABI), prepared them for mass spectrometric analysis and analyzed them as described ${ }^{1}$. We analyzed $33 \%$ of the avidinpurified peptides by $\mu \mathrm{LC}-\mathrm{MS} / \mathrm{MS}$ using an LCQ ion trap mass spectrometer (ThermoFinnigan). We identified peptides by searching MS/MS spectra against a yeast protein database using SEQUEST ${ }^{25}$. Quantification and data analysis were done using XPRESS and INTERACT computer programs, respectively ${ }^{6}$. All peptide identifications and quantifications were confirmed by manual inspection of the data. Abundance ratios were normalized to adjust for any systematic error due to sample handling. For the YDR079c-a-FLAG immunoprecipitation, we did this by generating a ratio distribution from the logarithm (base 10) of all peptide ratios and fitted with a normal distribution of the form $N(\lg r)=A^{*} \exp \left[-\left(\lg r / r_{0}\right)^{2} / 2 \sigma^{2}\right]$ in which $A=18.87, r_{0}=1.362$ and $\sigma=0.104$ (ref. 7). All protein ratios were then normalized by $r_{0}$. The corresponding standard deviations were propagated from the original standard deviations and the fitting error of $r_{0}$ (ref. 26). For a protein of ratio $r_{\mathrm{p}}$ and standard deviation $\Delta r_{\mathrm{p}}$, we calculated a $P$ value with the formula $P=\operatorname{erfc}\left[\left|\lg r_{\mathrm{p}} / r_{0}\right|\right.$ $/ \sqrt{ }\left(2\left(\Delta \lg r_{\mathrm{p}}^{2}+\sigma^{2}\right)\right]$, in which $\Delta \lg r_{P=\Delta} r_{\mathrm{p}} / r_{\mathrm{p}} \ln 10$.

URLs. The software AnalyzerDG is available at http://molecularware.com/analyzerdg.htm. In vitro transcription reaction methods are available at http://www.fhcrc.org/labs/hahn/.

GEO accession number. GSE1365.

Note: Supplementary information is available on the Nature Genetics website.

\section{ACKNOWLEDGMENTS}

We thank P. Mallick and B. Marzolf for help with data analysis, E. Schiebel for the pYM plasmids, T. Tsukiyama for pUG6 and p3FLAGKanMX plasmids, S. Buratowski for antibodies to TFB3 antibodies and yeast strains and Y. Chi, T. Krumm and N. Yudkovsky for critical reading of the manuscript. This work was supported by grants from the US National Cancer Institute and the National Institutes of Health Research Resource Center, by federal funds from National Heart, Lung, and Blood Institute, by a US National Institutes of Health contract and by a postdoctoral fellowship from the National Institutes of Health to J.A.R. Partial funding for this work came from a gift from Merck and Company to the Institute for Systems Biology.

\section{COMPETING INTERESTS STATEMENT}

The authors declare that they have no competing financial interests.

Received 13 February; accepted 25 May 2004

Published online at http://www.nature.com/naturegenetics/

1. Ranish, J.A. et al. The study of macromolecular complexes by quantitative proteomics. Nat. Genet. 33, 349-355 (2003).

2. Giglia-Mari, G. et al. A new, tenth subunit of TFIIH is responsible for the DNA repair syndrome trichothiodystrophy group A and stabilizes TFIIH. Nat. Genet. advance online publication, 27 June 2004 (doi:10.1038/ng1387)

3. Cenkci, B., Petersen, J.L. \& Small, G.D. REX1, a novel gene required for DNA repair. J. Biol. Chem. 278, 22574-22577 (2003).

4. Svejstrup, J.Q., Vichi, P. \& Egly, J.M. The multiple roles of transcription/repair factor TFIIH. Trends Biochem. Sci. 21, 346-350 (1996).

5. Matsui, P., DePaulo, J. \& Buratowski, S. An interaction between the Tfb1 and SsI1 subunits of yeast TFIIH correlates with DNA repair activity. Nucleic Acids Res. 23 767-772 (1995).

6. Han, D.K., Eng, J., Zhou, H. \& Aebersold, R. Quantitative profiling of differentiationinduced microsomal proteins using isotope-coded affinity tags and mass spectrometry. Nat. Biotechnol. 19, 946-951 (2001).

7. Li, X.J., Zhang, H., Ranish, J.A. \& Aebersold, R. Automated statistical analysis of protein abundance ratios from data generated by stable-isotope dilution and tandem mass spectrometry. Anal. Chem. 75, 6648-6657 (2003).

8. Feaver, W.J. et al. Genes for $\mathrm{Tfb} 2$, $\mathrm{Tfb} 3$, and $\mathrm{Tfb} 4$ subunits of yeast transcription/repair factor IIH. Homology to human cyclin-dependent kinase activating kinase and IIH subunits. J. Biol. Chem. 272, 19319-19327 (1997).

9. Svejstrup, J.Q., Feaver, W.J., LaPointe, J. \& Kornberg, R.D. RNA Polymerase transcription factor IIH holoenzyme from yeast. J. Biol. Chem. 269, 28044-28048 (1994).

10. Sung, P., Guzder, S.N., Prakash, L. \& Prakash, S. Reconstitution of TFIIH and requirement of its DNA helicase subunits, Rad3 and Rad25, in the incision step of nucleotide excision repair. J. Biol. Chem. 271, 10821-10826 (1996).

11. Svejstrup, J.Q. et al. Different forms of TFIIH for transcriptional and DNA repair: holoTFIIH and a nucleotide excision repairosome. Cell 80, 21-28 (1995).

12. Adamczewski, J.P. et al. MAT1, cdk7 and cyclin $\mathrm{H}$ form a kinase complex which is UV light-sensitive upon association with TFIIH. EMBO J. 15, 1877-1884 (1996).

13. Vermeulen, W. et al. Sublimiting concentration of TFIIH transcription/DNA repair factor causes TTD-A trichothiodystrophy disorder. Nat. Genet. 26, 307-313 (2000).

14. Tirode, F., Busso, D., Coin, F. \& Egly, J.M. Reconstitution of the transcription factor TFIIH: assignment of functions for the three enzymatic subunits, XPB, XPD, and cdk7. Mol. Cel/ 3, 87-95 (1999).

15. Knop, M. et al. Epitope tagging of yeast genes using a PCR-based strategy: more tags and improved practical routines. Yeast 15, 963-972 (1999).

16. Guldener, U., Heck, S., Fielder, T., Beinhauer, J. \& Hegemann, J.H. A new efficient gene disruption cassette for repeated use in budding yeast. Nucleic Acids Res. 24 2519-2524 (1996).

17. Gelbart, M.E., Rechsteiner, T., Richmond, T.J. \& Tsukiyama, T. Interactions of Isw2 chromatin remodeling complex with nucleosomal arrays: analyses using recombinant yeast histones and immobilized templates. Mol. Cell. Biol. 21, 2098-2106 (2001).

18. Ausubel, F.R. et al. Introduction of a point mutation by sequential PCR steps. in Current Protocols in Molecular Biology (John Wiley \& Sons, New York, 2002).

19. Ideker, T., Thorsson, V., Siegel, A.F. \& Hood, L.E. Testing for differentially-expressed genes by maximum-likelihood analysis of microarray data. J. Comput. Biol. 7, 805-817 (2000).

20. Ideker, T. et al. Integrated genomic and proteomic analyses of a systematically perturbed metabolic network. Science 292, 929-934 (2001).

21. Iyer, V. \& Struhl, K. Absolute mRNA levels and transcriptional initiation rates in Saccharomyces cerevisiae. Proc. Natl. Acad. Sci. USA 93, 5208-5212 (1996).

22. Kang, J.J., Auble, D.T., Ranish, J.A. \& Hahn, S. Analysis of the yeast transcription factor TFIIA: distinct functional regions and a polymerase II-specific role in basal and activated transcription. Mol. Cell. Biol. 15, 1234-1243 (1995).

23. Ranish, J.A., Yudkovsky, N. \& Hahn, S. Intermediates in formation and activity of the RNA polymerase II preinitiation complex: holoenzyme recruitment and a postrecruitment role for the TATA box and TFIIB. Genes Dev. 13, 49-63 (1999).

24. Ranish, J.A. \& Hahn, S. The yeast general transcription factor TFIIA is composed of two polypeptide subunits. J. Biol. Chem. 266, 19320-19327 (1991).

25. Eng, J.K., McCormack, A.L. \& Yates, J.R. An approach to correlate tandem mass spectral data of peptides with amino acid sequences in protein databases. J. Am. Soc. Mass Spectrom. 5, 976-989 (1994)

26. Taylor, J.R. An Introduction to Error Analysis: The Study of Uncertainties in Physical Measurements (University Science Books, Sausalito, 1997).

27. Reddy, P. \& Hahn, S. Dominant Negative Mutations in Yeast TFIID Define a Bipartite DNA-Binding Region. Cel/ 65, 349-357 (1991). 